\title{
A Review of IoT Technology for the Connected Autonomous Vehicles Ecosystem
}

\author{
Nishant Sharma and Parveen Sultana Habibullah* \\ School of Computer Science and Engineering, Vellore Institute of Technology, Vellore 632014, \\ Tamil Nadu, India
}

("Corresponding author’s e-mail: hparveensultana@vit.ac.in)

Received: 8 November 2020, Revised: 1 June 2021, Accepted: 8 June 2021

\begin{abstract}
Connected Autonomous Vehicles Ecosystem (CAVE) is fast becoming a reality. This paper reviews various architectures that leverage IoT technology to progressively create a system of interconnected Autonomous Vehicles (AV) that is effective, robust and secure. Accurate and fast perception of the external environment is key to movement of AVs in CAVE. IoT technology through sensors and internetworking can help an AV to do reliable perception. State of the art methods for perception of AVs in CAVE are reviewed. Self-awareness is another important aspect. IoT and Artificial Intelligence (AI) can come together to assist an AV in self-diagnosing anything abnormal. One such use case is presented for onboard diagnostics in an AV. Accurate AI is key to making effective strategies and to take control actions with error feedbacks that carefully maneuver an AV from its source to destination. The transition from legacy vehicles to CAVE requires AI methods that combat threats and have effective overall movement in mixed mode traffic systems (MMTS). Strategies for effective movement in MMTS are presented.
\end{abstract}

Keywords: IoV, Connected cars, Autonomous vehicles, Cognitive IoV, Artificial Intelligence

\section{Introduction}

The number of road accidents in India for the year 2015 are provided in [1]. It is claimed that drivers are held responsible for $78 \%$ of the accidents. The mistakes usually committed by human drivers are over speeding, driving under the influence of alcohol or drugs and hit and run. These accidents may be fatal to either the driver or others on the road. Although online articles like [2] paint a gloomy picture of the possibility of successful deployment of AVs on Indian roads, AVs may provide an effective solution and bring down the number of road fatalities in India due to chaotic traffic, poor infrastructure and bad traffic habits. The technology that underpins AVs is the Internet of Things. A lot of ongoing research is exploring the combination of these ideas under the headings of Internet of Vehicles (IoV), Connected Cars and Connected Autonomous Vehicles. There is also a dedicated effort by companies like Google and Tesla to bring reliable AVs into the world as quickly as possible.

Internet of Things, which can be defined as the interconnection of everyday objects into a network such that they have the capability to communicate information amongst each other, has found many applications in the modern world. It also has its fair share of challenges [3]. IoT end nodes are constrained by memory and battery power. There are timing constraints, unreliable networks, latency issues, security and privacy concerns. IoT itself is constantly under the microscopic lens of the research community. Simultaneous research is also ongoing to create reliable CAVE.

Waymo, a subsidiary of Alphabet Inc. (the parent company of Google), states its motivation for building AVs as making driving safe and enjoyable while preventing loss of life due to traffic crashes (which were 1.35 million worldwide in 2016). The process flow for Waymo's AVs is as follows. First, these vehicles are equipped with high-definition 3D maps that provide a detailed profile of the vehicular environment. Various sensors attached to a vehicle perceive constantly the change in state of other vehicles around it on its path. These sensors perceive the motion of pedestrians, perceive state changes for traffic lights and much more to provide the vehicle with a good sense of environmental awareness. The speed and trajectory of all moving objects in the environment of a vehicle along with the long-term objective of reaching the destination are utilized by the accompanying software to identify the most 
appropriate next action for the vehicle. The accompanying software takes the control action and then repeats the process flow to maneuver the vehicle carefully to the destination. In [4], the authors coined the term Quality of IoT Experience (QoIoT) for AVs which combines the traditional quality of experience model centered around the end users with a quality of experience model aimed at evaluating the machine performance in terms of defined business metrics. Minovski et al. [4] also discusses the challenges of human-AV interaction where humans can affect and alter functionalities of AVs and an AV can impact the quality of experience of a human end-user.

Connected cars is a paradigm where the vehicles, whether fully autonomous or not, communicate with each other and relay information. Connected cars paradigm is useful because it increases the situational awareness of a platoon of vehicles [5]. The overall perception of an AV in CAVE can be improved by augmenting its sensor-based perception of the external environment with a shared perception with other AVs in its network. For example, an AV halted by a broken bridge and blocking the view of a trailing AV can now relay its [the former's] perception information to the latter thus improving the situational awareness of the trailing AV. Other benefits of CAVE are reduced traffic congestion and lower $\mathrm{CO}_{2}$ emissions. This is due to better global management of traffic through effective decision making by utilizing the real time information provided by these vehicles to the Cloud [6]. Robust and reliable CAVE may be considered as one of the concluding milestones.

The Society of Automation Engineers (SAE) enlists 6 levels of driver automation. Level 0 has no automation and the human driver does all the driving. Level 1 has an Advanced Driver Assistance System (ADAS) which assists the human driver in either one of the following: The job of steering the vehicle or the job of braking and acceleration but it cannot manage multiple control actions simultaneously. Inevitably, the human is the main driving agent. Level 2 starts to incorporate automation in its essence into the ADAS. A Level 2 system can make more than 1 control action simultaneously. The human driver must still be available at all times to take control of the vehicle even when the ADAS is active. Some control actions are performed by the ADAS to assist the human driver while most control actions are still the responsibility of the human driver. The human driver must also monitor the outside environment at all times. Level 3 takes the automation a step further. Here, the ADAS can be relied upon to drive in certain environments. However, the ADAS may relinquish control to the human driver at a moment's notice if the favorable conditions for ADAS appear to be violated. Under unfavorable conditions, the human driver does all the driving. For Level 4 systems, a high level of automation is necessary. For environments in which the Level 4 ADAS has been trained, it is self-sufficient and does not relinquish control to the human driver. A human driver must take over only when the ADAS is unfamiliar with a particular environment. At Level 5 and at full automation, the AV is self-sufficient and drives itself under all circumstances and for all possible environments.

National Highway Traffic Safety Administration (NHTSA) enlists 5 eras of safety (calendar years) that can be mapped to the $6 \mathrm{SAE}$ Levels of Automation. At SAE Level 0 and for 0 automation, the automobile is expected to have safety features such as seat belts and anti-locking brakes. NHTSA envisions this era to have lasted between 1950s and 2000. For SAE Level 1 and for driver assistance, the automobile requires not only the advanced safety features such as blind spot warning, forward collision warning and lane departure warning but also the advanced driver assistance features such as automatic emergency features and lane centering assist. This period is classified between the years 2000 and 2016. At Level 2 and for partial automation, the automobile requires at least partially automated safety features such as traffic jam assist and self-parking. It is expected that the partially automated safety features shall be integrated into most of the mainstream automobiles by the year 2025. Levels 3 - 5 that enable higher levels of automation expect fully automated safety features to be integrated into an automobile. The research and implementation phases in this era are expected to begin by the year 2025. Figure 1 summarizes the discussion so far.

Yuen et al. [7] presents a discussion on how acceptance of AVs by the public is the key to the success of CAVE. Various constructs and measurement items have been identified such as ease of usage, ethics, cost of ownership and reliability of AVs. Maurer et al. [8] does a comprehensive presentation of the importance of encoding human understood ethics into an AV. It also discusses implementing such ethics. Siegel et al. [9] comprehensively presents the technological challenges for application deployment in CAVE. The concerns illustrated are network related performance issues, design of efficient and scalable protocols and standards for Vehicle to Everything (V2X) paradigm (discussed later), the privacy and security concerns. The challenges related to vehicle design are reliable perception, choosing an appropriate mode of communication and concerns related to hardware hijacking. In [9], challenges related to handling big data and providing appropriate infrastructure are also discussed. 


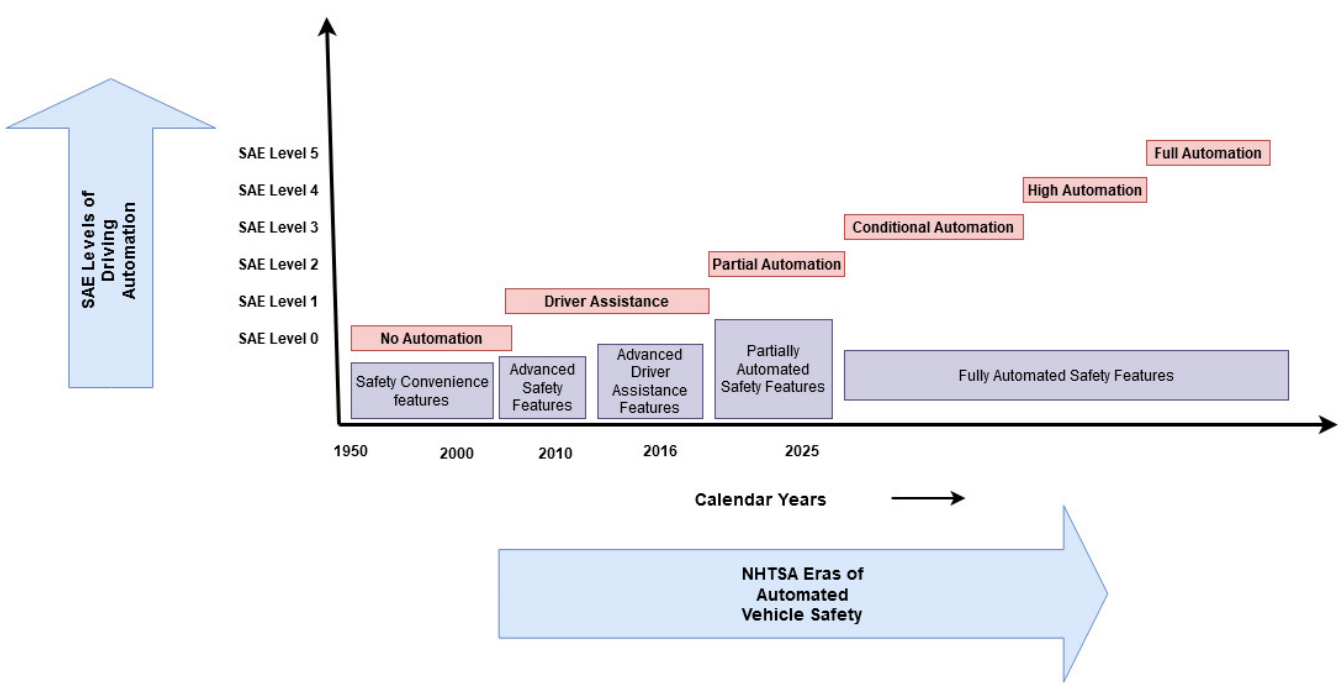

Figure 1 Stages of Automation and Eras of Automated Vehicle Safety as given by SAE and NHSTA, respectively.

\section{Evolution of architectures for CAVE}

The basic architecture of IoV is 3 layered: The 3 Cs - 1) Client, 2) Connection and 3) Cloud [5]. The Client layer is composed of all sensors that are present in a vehicle. The connection layer is responsible for facilitating the inter-vehicular communication and the communication of a vehicle with its environment. The terminologies used to describe these interactions are V2V (Vehicle to Vehicle), V2I (Vehicle to Infrastructure) which encompasses communications such as V2R (Vehicle to Roadside), V2P/V2H (Vehicle to Pedestrian/Vehicle to Humans), V2S/V2D (Vehicle to Sensors/Vehicle to Device) and (V2G) Vehicle to Grid. V2V and V2I are contained in V2X (Vehicle to Everything) paradigm [10]. The Cloud layer provides infotainment (both information and entertainment) framework for applications that run on an AV.

Kaiwartya et al. [11] presents a 5 layered architecture for IoV. The 5 layers are 1) Perception layer, 2) Coordination layer, 3) Artificial intelligence layer, 4) Application layer and 5) Business layer. The Perception layer is similar to the Client layer in [5]. The Coordination layer is similar to the Connection layer. However, the Cloud layer is now diversified into 3 layers: 1) Artificial intelligence layer, 2) Application layer and 3) Business layer. Artificial intelligence layer is responsible for making critical decisions based on processing and analyzing data that is stored on the Cloud. Application layer provides framework for applications that range from information to entertainment. Business layer provides framework for business models and services. These models and services may be created based on the foresight provided by data which is stored, processed and analyzed on the Cloud.

Chen et al. [12] provides a similar 5 layered architecture to [11] and calls it Cognitive IoV. The layers are named as 1) Sensing layer, 2) Communication layer, 3) Cognition layer, 4) Control layer and 5) Application layer. It is noted that the Cognition layer assumes responsibility similar to Artificial intelligence layer in [11]. Similarly, the other layers find resemblance.

Contreras-Castillo et al. [13] goes a step further to provide a 7 layered architecture for IoV. In [13], the authors identified weakness in the 3 and 5 layered architectures discussed above and argued for the integration of security mechanisms into the functionality of each layer. Contreras-Castillo et al. [13] argues for the pre-processing of data in order to avoid network congestion in the vehicular inter-network. Contreras-Castillo et al. [13] also argues that a choice among various networking methods should be available for an AV to communicate with other AVs or to access infotainment services based on the need of the application and the strengths of a networking method; the term that they coined is communication intelligence. Additionally, the authors of [13] added audio, visual and haptic interfaces for interaction of humans with the AV. The 7 layers named by them are: 1) User-vehicle interface layer, 2) Data acquisition layer, 3) Data filtering and pre-processing layer, 4) Communication layer, 5) Control and management layer, 6) Processing layer and 7) Security layer. 


\section{Perception in CAVE}

If the 3 Cs architecture was to be crudely reduced to 3 functionalities: 1) Sensory perception, 2) Inter-vehicular communication and 3) Applications; it is both the sensory perception of an AV and cooperative perception with other AVs in CAVE through inter-vehicular communication that combines to form the overall perception of the AV. In this section, we will review both of these ways of perception.

\section{Sensors}

An AV consists of many sensors that individually obtain pieces of information about its environment and which when combined together, understood and inferred from, create an awareness in the AV regarding its physical environment. Before we discuss about various sensors available today for the realization of AVs, it is beneficial to summarize the parameters that are desired for a sensor for its applicability in AVs [14,15]. Table 1 reviews and discusses these parameters.

\section{Perception of the external environment}

The type of sensors that are useful for perception of external environment in an AV and their respective benefits are presented in Table 2. It is preferrable to fuse measurements obtained from multiple sensors as opposed to having only 1 dedicated sensor in order to increase the reliability of the measurement. Using sensor fusion avoids the possibility of faulty measurements dictating the perception of an AV. A multi-sensor fusion system can utilize sensed data from many different sensors to create a reliable measurement of the observed property. Multiple sensors of similar type can also be used to increase the reliability of 1 type of measurement. Realpe et al. [16] presents a fault tolerant perception system for AV vision where the vision sensors could either be of a similar or a different type. The idea is to create an architecture that reduces the impact of a faulty sensor on the final agreed value of the observed property. It does so by entering the vision system into a tolerated error state from where an attempt at recovery can be made to avoid a complete system failure.

Table 1 Desired parameters for sensors in CAVE.

\begin{tabular}{clcc}
\hline Parameter & \multicolumn{1}{c}{ Definition } & Desirability \\
\hline Accuracy & The difference between the actual value $(\mathrm{A})$ of a property being & High \\
& $\begin{array}{l}\text { measured and the value obtained from the sensor }(\mathrm{O}) \text {; the accuracy is } \\
\text { usually expressed as a percentage of the full-scale range/full range }(\mathrm{F}) \text { as } \\
\\
(|\mathrm{A}-\mathrm{O}| / \mathrm{F} \times 100 \%) .\end{array}$
\end{tabular}

Error How much does the measurement of a property obtained by a sensor deviates from the measurement that would be obtained from an ideal sensor under similar conditions.

Hysteresis Two measurements M1 and M2 are taken for the same input value in a series of increasing and decreasing input values respectively. Hysteresis is $|\mathrm{M} 1-\mathrm{M} 2|$, where $|\mathrm{x}|$ is the absolute value of $\mathrm{x}$.

Non- Linearity A straight line is attempted for the values of a property obtained from a sensor over a desired range that best fits all those values. How much the measured output values from the sensor deviate from the best fit line is described as non-linearity.

Offset How far the measured value of a property is from the actual value is called the offset. However, the offset is constant along all measured outputs and is not an error in scaling.

Range The closed interval of the minimum and the maximum values of the measurand that can be measured by a sensor.

Low

Low

Resolution

The smallest change $\delta$ in the measured property that is recognized by a sensor. 


\begin{tabular}{clc}
\hline Parameter & \multicolumn{1}{c}{ Definition } & Desirability \\
\hline Sensitivity & $\begin{array}{l}\text { The change in output value } \delta \text { resulting from the change in input value } \delta \text { i } \\
\text { of the measurand taken as a ratio } \delta \text { o/ } \delta \text { i. }\end{array}$ & High \\
Smartness & $\begin{array}{l}\text { The steps such as integration of microcontrollers that enhance the } \\
\text { capabilities of a sensor such that it can do additional logistical } \\
\text { information processing and thus improve the quality of provided output. }\end{array}$ & High \\
Stability & $\begin{array}{l}\text { Remains reliable for the duration of an experiment or for a reasonably } \\
\text { long period of time. }\end{array}$ & High \\
Warmup time & $\begin{array}{l}\text { The time required by a sensor to reach its stable state after it is switched } \\
\text { on. }\end{array}$ & Low \\
\hline
\end{tabular}

\section{Vehicle to everything paradigm}

In Figure 2, a pictorial representation of Vehicle to Everything (V2X) concept is presented. It presents various types of communication that can happen between an AV and its environmental objects. Zhou et al. [17] presents a discussion on how to move from V2X to IoV. In [17], the authors have discussed challenges and opportunities relating to both big-data driven IoV and Cloud/Edge based IoV.

Table 2 Sensors used for perception of the external environment.

\begin{tabular}{|c|c|}
\hline Type of Sensor & Sensor Benefits \\
\hline Camera & Lane detection. Staying within lane and departure from the lane. \\
\hline RADAR (Radio Detection and Ranging) & $\begin{array}{l}\text { Near and far obstacle detection using radio waves. } \\
\text { Detection of an object's distance and speed. } \\
\uparrow \text { Cost and reliability. } \\
\downarrow \text { Not as accurate as LiDAR. }\end{array}$ \\
\hline LiDAR (Light Detection and Ranging) & $\begin{array}{l}\text { Obstacle detection using light. } \\
\uparrow \text { Accuracy. Speed of operation. Better at creating accurate maps of the } \\
\text { environment. } \\
\downarrow \text { Cost. }\end{array}$ \\
\hline Far Infra-Red Thermal Sensor & Detecting objects in low lighting environment (night vision). \\
\hline Ultrasonic Sensor & $\begin{array}{l}\text { Obstacle detection using SONAR (Sound Navigation and Ranging). } \\
\text { Mostly used in parking applications. } \\
\uparrow \text { Cost. } \\
\downarrow \text { Measurements impacted by environmental factors. }\end{array}$ \\
\hline
\end{tabular}

$\uparrow:$ Advantages; $\downarrow$ : Disadvantages

Ning et al. [18] proposes an IoV architecture for CAVE powered by Edge. Road side units are equipped with edge servers where computation offloading can be done by an AV. If a computation exceeds the capability of an edge server, then it may be offloaded to the Cloud. Infotainment services can be delivered from the Cloud to an AV using efficient networking services. One of the ways in which the various infotainment services can be consumed by an AV is demonstrated in [19]. Hassani et al. [19] provides a use case for Context as a Service for IoT based parking applications in AVs. All the real time data available regarding parking slots for a location is used to provide an empty parking slot to an AV. Such a service may employ a shared global vehicular Cloud. 
Two technologies contending to be de-facto standards for V2X communication are the Dedicated Short Range Communication (DSRC) and 5G. 5G technology is in its infancy and it follows LTE technology. DSRC is standardized by IEEE as $802.11 \mathrm{p}$ for wireless communication in vehicular environments. LTE and $5 \mathrm{G}$ are standardized by $3^{\text {rd }}$ Generation Partnership Project (3GPP). A chart comparing DSRC and LTE is shown as Table 3. Vendors for infotainment services in CAVE may choose a hybrid solution that uses both DSRC and LTE (or 5G) for their respective strengths.

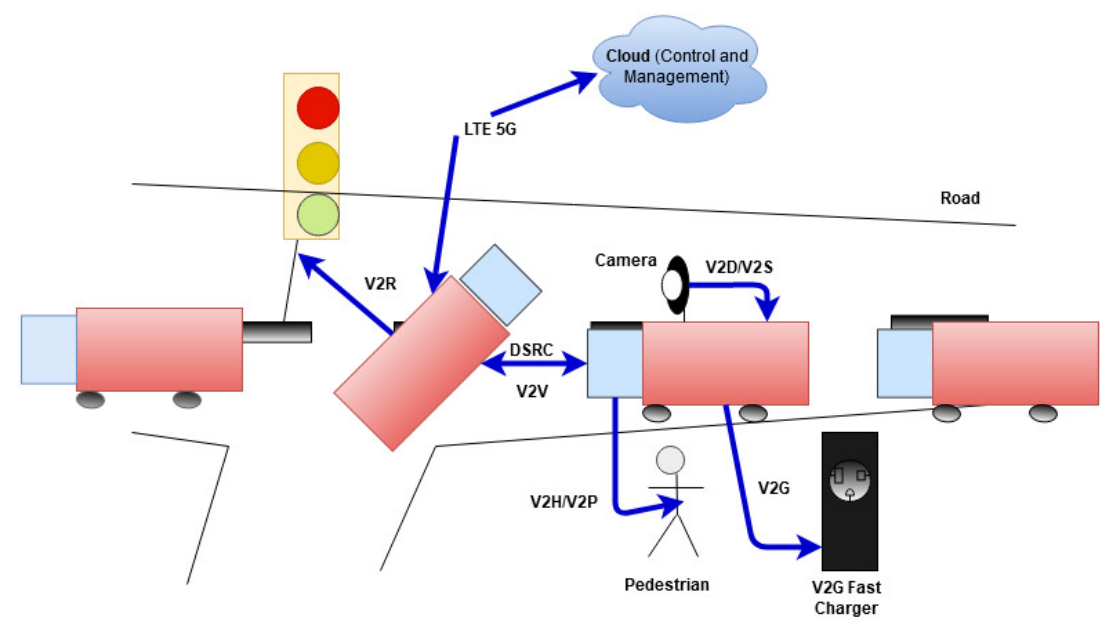

Figure 2 Vehicle to Everything (V2X) paradigm.

Table 3 Comparison of 802.11p and LTE based Wireless Communication Technology.

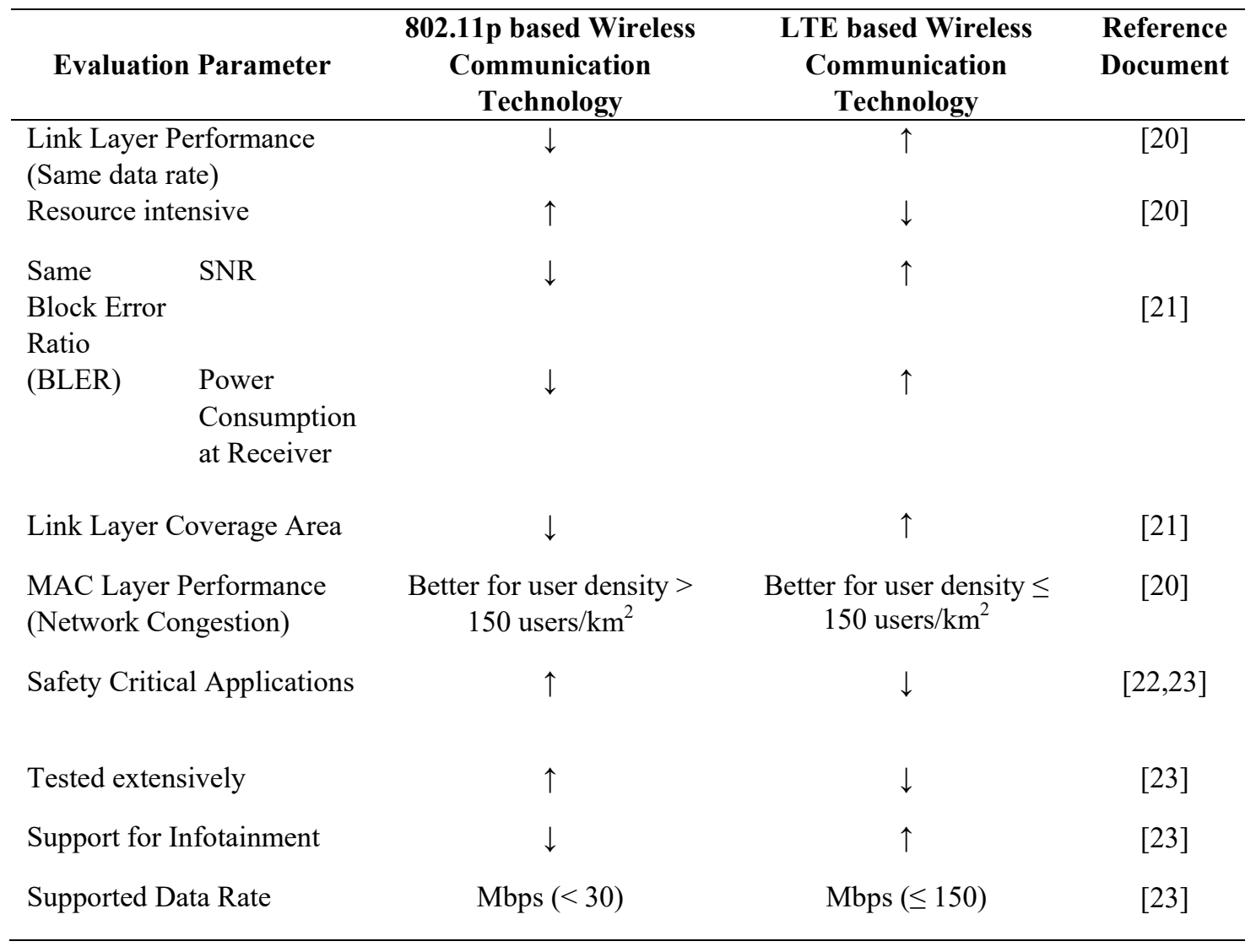

$\uparrow:$ In favor of; $\downarrow$ : Against. 


\section{Inter-vehicular network based perception}

AVs in CAVE can enhance each other's perception by sharing information among each other. Two AVs within a bounded distance can share information between each other or an AV can pass information to the shared Cloud which can be used for global analytics. This cooperative perception increases the situational awareness for an AV in CAVE. An example of situational awareness for an AV in CAVE can be to know about a broken bridge or a scheduled road maintenance en route to the AV's destination for it to plan an alternate route without losing a lot of time. Cooperative perception will also reduce hostility between AVs in CAVE. An AV halted by a genuine environmental factor (such as scheduled road maintenance) can share its perception information to another AV whose view the former might obstruct. This will not only update the perception information for the latter AV but also inform the passengers about the current situation. The shared information must preserve the respective frames of reference and coordinate points for the environmental objects that are perceived [24]. An open-source V2X cooperative perception system integrating the individual perception of an AV obtained from its software: Autoware with an open-source experimental and prototyping software for cooperative Intelligent Transport Systems (ITS): OpenC2X is presented in [25]. Open-source implementations are more reliable since they are in public domain and are accessible to all. Experts can find flaws and attempt optimizations that are open to comments and criticism. Understanding and discussing such systems in public domain will help CAVE find a faster acceptance in the real world.

\section{Localization}

Localization is the ability of an AV to pin point itself. For an AV in CAVE, the localization process is as shown in Figure 3. GPS.gov states that GPS enabled devices in open environments are accurate to a radius of $4.9 \mathrm{~m}$. Error in localization of an AV can prove to be fatal. If the location of an AV is known, a high-definition map can easily be created for its environment. If a high-definition map for a vehicular environment is present, systems like Global Navigation Satellite System (GNSS) of which Global Positioning System (GPS) is 1 component, Inertial Sensors (IMUs), Camera, RADAR and LiDAR can be utilized by an AV's software to localize the AV on the high-definition map with high accuracy. Simultaneous Localization and Mapping (SLAM) [26] is a more sophisticated technique that aims to combine both of the above processes into 1. In SLAM a high-definition map of the vehicular environment is created while trying to approximate the location of an AV on that map. The shared cooperative perception discussed earlier can also be used to localize an AV from a frame of reference of another AV in its neighborhood. An AV that has successfully localized itself can pass its localization information (and the respective coordinate frames of reference) to another AV in its neighborhood and reduce the computational overhead of the latter AV in localizing itself.

\section{On-board diagnostics}

In addition to having sensors that provide accurate measurements for perception in an $\mathrm{AV}$, various sensors must also be installed onto an AV that help in its maintenance and to avoid complete system failure. An artificial intelligence layer can use the data available from internal sensors to predict the possibility of a system failure. If a failure is imminent, proper messages and notifications can be sent to the concerned authorities. Table 4 presents an overview of various sensors that can help in onboard diagnostics in an AV.

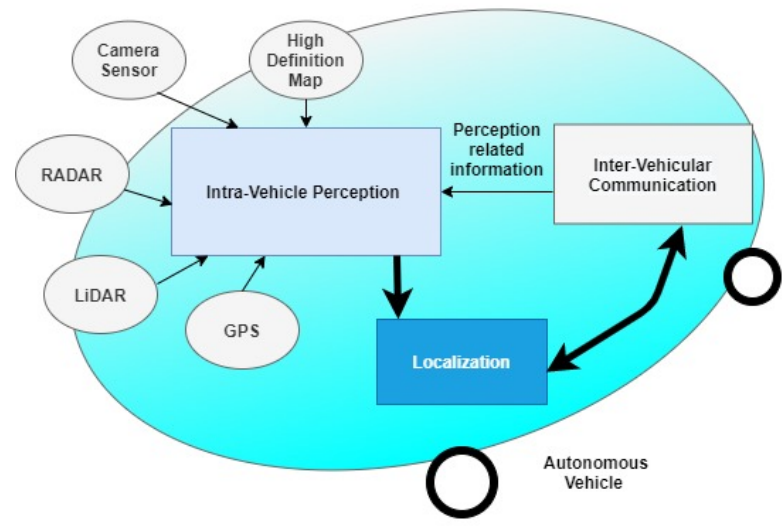

Figure 3 Localization in CAVE. 
Table 4 Sensors for onboard diagnostics in an AV.

\begin{tabular}{|c|c|c|}
\hline Sensor Type & $\begin{array}{c}\text { Sensor } \\
\text { Position }\end{array}$ & Sensor Application \\
\hline $\begin{array}{l}\text { Mass flow Sensor } \\
\text { (MAF) }\end{array}$ & Engine & $\begin{array}{l}\text { MAF measures mass flow rate of air to a fuel injected AV engine. } \\
\text { Information obtained through an MAF sensor assists in delivering the } \\
\text { correct fuel mass to the AV engine. }\end{array}$ \\
\hline $\begin{array}{l}\text { Manifold Absolute } \\
\text { Pressure Sensor } \\
\text { (MAP) }\end{array}$ & Engine & $\begin{array}{l}\text { MAP measures the manifold pressure of an AV engine. This measured } \\
\text { value is used to calculate the air density and to determine the mass } \\
\text { flow rate of air to the fuel injected AV engine. MAP is used to } \\
\text { calculate the fuel for optimum combustion at a given time. }\end{array}$ \\
\hline $\begin{array}{l}\text { Crankshaft } \\
\text { Position Sensor } \\
\text { (CPS) }\end{array}$ & Engine & $\begin{array}{l}\text { CPS is connected to an engine's crankshaft. It measures the rotational } \\
\text { speed of the crankshaft. Information obtained from CPS assists in } \\
\text { regulating fuel injection based on the observed engine speed. }\end{array}$ \\
\hline $\begin{array}{l}\text { Engine Knocking } \\
\text { Detection Sensor } \\
\text { (EKDS) }\end{array}$ & Engine & $\begin{array}{l}\text { EKDS alerts the engine computer if the fuel starts to burn before the } \\
\text { "right" time, or if the combustion is abnormal. Monitoring through } \\
\text { EKDS can be useful in minimizing damage to an engine's internal } \\
\text { modules. A commonly used EKDS sensor is a piezoelectric sensor } \\
\text { which is programmed to detect imperceptible sounds of knocking in } \\
\text { case of abnormal combustion for appropriate action by the engine } \\
\text { computer. }\end{array}$ \\
\hline $\begin{array}{l}\text { Oxygen Sensor } \\
\text { (OS) }\end{array}$ & Engine & $\begin{array}{l}\text { OS measures oxygen content in the exhaust gases. This value of } \\
\text { oxygen content can be used to calculate the air to fuel ratio. The air to } \\
\text { fuel ratio can be adjusted in order to optimize fuel consumption. }\end{array}$ \\
\hline $\begin{array}{l}\text { Fuel Temperature } \\
\text { Sensor (FTS) }\end{array}$ & Engine & $\begin{array}{l}\text { FTS measures the amount of fuel injection into an engine so that the } \\
\text { emissions and fuel economy are controlled and are in check. }\end{array}$ \\
\hline $\begin{array}{l}\text { Throttle Position } \\
\text { Sensor (TPS) }\end{array}$ & Engine & TPS is used to measure the air intake of an AV engine. \\
\hline $\begin{array}{l}\text { Engine Coolant } \\
\text { Temperature } \\
\text { Sensor (ECTS) }\end{array}$ & Engine & $\begin{array}{l}\text { ECTS measures the coolant temperature. Information obtained from } \\
\text { ECTS can be used to regulate fuel injection. }\end{array}$ \\
\hline $\begin{array}{l}\text { Valve Pressure } \\
\text { Sensors (VPS) }\end{array}$ & Tire & $\begin{array}{l}\text { Usually, a battery driven VPS is placed on the inside of the valve of an } \\
\text { AV tire. VPS measures the pressure of the tire directly. }\end{array}$ \\
\hline $\begin{array}{l}\text { Wheel Speed } \\
\text { Sensors (WSS) } \\
\text { Accelerometer } \\
\text { Sensors (AS) }\end{array}$ & Tire & $\begin{array}{l}\text { WSS and AS provide measurements which are used indirectly (using } \\
\text { software) to monitor the pressure of an AV tire. }\end{array}$ \\
\hline
\end{tabular}

\section{Proposed methodology for onboard diagnostics in AV}

Various sensors measure data over time and this data is made available to a central processing unit inside an $\mathrm{AV}$. In the proposed methodology, the onboard diagnostics is achieved by doing anomaly detection for measurements obtained from AV sensors. A hot time-frame (window) is identified where multiple sensors report anomalous measurements. This identified time-frame is considered for necessary intervention in the AV. The process for anomaly detection for onboard diagnostics in an AV takes the following steps: 


\section{Step 1}

Attributes in the dataset containing measurements from various sensors over time may have correlation between them. A threshold value is defined beyond which 2 attributes are considered strongly correlated whether in a positive or negative way. Pairwise correlation is calculated for all pairs of attributes in the dataset except the $1^{\text {st }}$ attribute representing time. If the pairwise correlation of any pair is found to go beyond the threshold value, then the $2^{\text {nd }}$ attribute is dropped. This step is repeated until no attributes remain in the dataset that have a correlation beyond the threshold value.

\section{Step 2}

A list of (time, attribute value) tuples is created for all rows of an attribute to keep track of the time value at which an attribute value was observed. Such a list is created for all uncorrelated attributes.

\section{Step 3}

Find the mean and standard deviation for attribute values in an attribute. Define lower bound for an attribute as $\lambda-\mu \times \sigma$, where $\lambda$ is the mean of the attribute, $\sigma$ is the standard deviation and $\mu$ is a tunable parameter. Similarly, define upper bound as $\lambda+\mu \times \sigma$. All values lower than the lower bound and greater than upper bound are anomalies. A list of tuples of anomalous values and the time at which they were observed is created for an attribute. This step is performed for all uncorrelated attributes.

\section{Step 4}

A scatter plot is created for an attribute with the $\mathrm{x}$ axis representing the time of observing an anomalous value and $\mathrm{y}$ axis representing the respective anomalous value. Such a plot is drawn for all uncorrelated attributes.

\section{Step 5}

A hot time-frame is highlighted where maximum number of uncorrelated attributes observed anomalous values.

This process of anomaly detection is applied on sample data 1a6bd1826bbed87ef815498494d52745 .csv available from [27]. Different approaches for anomaly detection on same dataset are also presented in [28]. For the sample run shown in Figure 4, the chosen value of threshold is 0.81 and $\mu$ is 1.99.

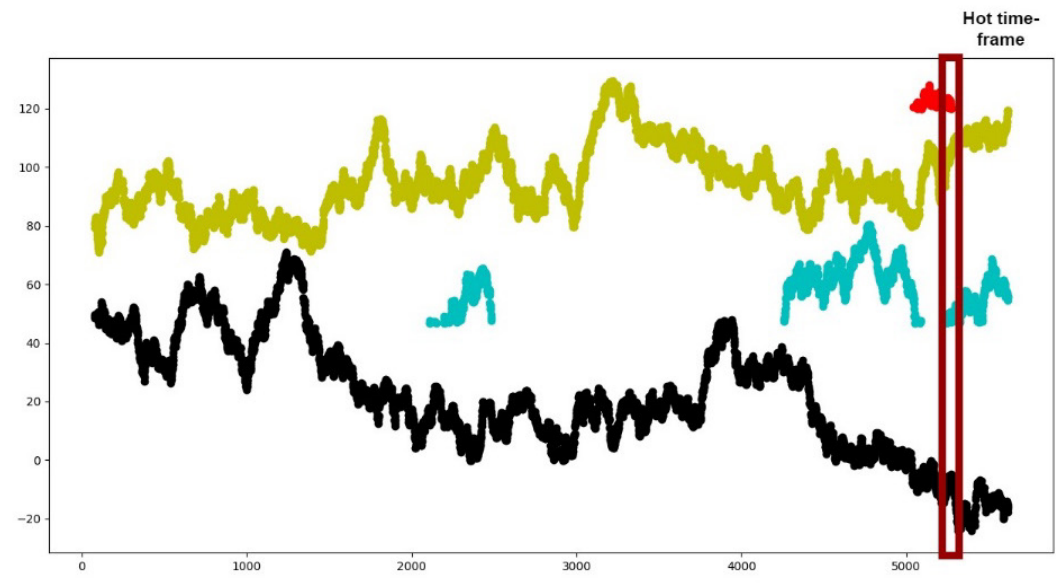

Figure 4 A hot time-frame is identified where maximum number of uncorrelated attributes observe anomalous values.

\section{Control actions for AV in CAVE}

Once the perception phase of an AV is complete, the artificial intelligence layer takes over. Approaches for AI in AV are discussed in [29]. The artificial intelligence layer does the strategic analysis. It does situation comprehension and devises a strategy for what route to take for the destination. It predicts how the traffic and environment are expected to behave during various points in time in the 
journey. It estimates the next action for an AV, the average speed with which to move, the blind spots to look out for, the expected time of arrival and much more. The process of perception and strategic planning is repeated and control actions are taken to carefully maneuver the AV to its destination. Errors in control action are provided as a feedback to the next phase of strategic planning. The process flow for control actions for an AV is reactive. A process flow for control actions for an AV adapted from [8] is shown in Figure 5.

Even when a vehicle is not fully autonomous, there are many parameters that can assist a human driver or a central processing unit inside a vehicle in making correct control decisions. Some of these are: 1) The speed of the tires and their angle of rotation on a desired turn, 2) The lateral and longitudinal acceleration of the wheels, 3) The force on and the state of the steering wheel, 4) The state of the gear and brake input and 5) The state of other assistive signals such as the turn signal, headlights and wipers during rain. Tracking these parameters can greatly enhance the system's reliability and reduce chances of a fatal accident.

\section{Members of CAVE}

The members of CAVE can be divided into 2 groups: The trustworthy entities and other entities.

\section{The trustworthy entities}

The trustworthy entities (TE) for an AV in CAVE include other AVs or RSUs that are certified by a recognized certifying authority. TEs include trustworthy technology providers such as cellular service providers for reliable infotainment access. TEs also include $3^{\text {rd }}$ party service providers that provide malware free services with policies ensuring privacy of data and secure access among others. Other AVs who have their certification expired but belong to a certified AV vendor may also qualify for a cautious trust. TEs will also include certified servicing centers for replacements of faulty parts and for maintenance of an AV.

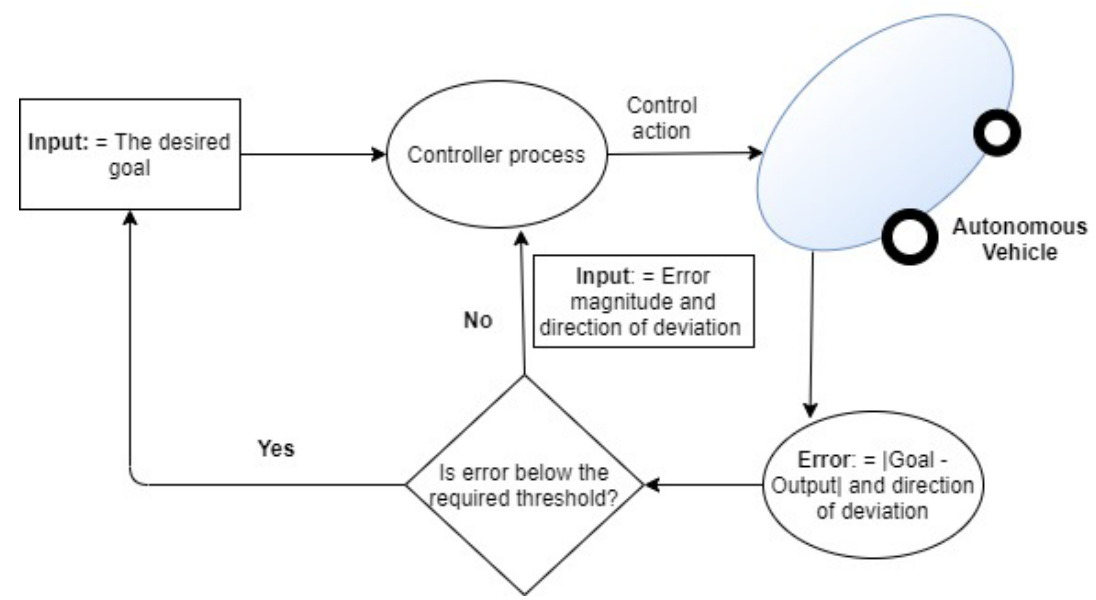

Figure 5 The reactive process flow of control actions for an AV.

\section{Other entities}

Other entities (OE) include natural entities in the CAVE that are unconnected such as a rock, a lake or a bridge. OEs also include legacy vehicles unconnected to CAVE. MMTS consisting of AVs, TEs and OEs will exist when there is transition from legacy unconnected vehicles to CAVE. Lima et al. [30] presents a threat model for malicious actors intending to harm an AV in MMTS. Lima et al. [30] enlists various types of threats in the threat model: 1) The threat to global communication, 2) Hijacking intervehicular communication, 3) Hardware or software hijacking of an AV, 4) Hijacking of RSUs and 5) Attack on authentication mechanisms between an AV and TEs. As perception ability increases for AVs through use of superior sensors or through better AI cognitive models, an AV may be able to predict a malicious attack on itself and take appropriate measures to block such an attack. AVs must have mechanisms coded in to deal with misuse cases such as a passenger sitting inside an AV and attempting to hijack it from inside. Self-preservation mechanisms against malicious attacks on AVs should be hard- 
coded to their artificial brains. For movement related strategies in MMTS, various ideas can be explored. Common wisdom dictates that an intelligent object on a path in an environment should only follow non intelligent objects. Another strategy could be to consider a system of 3 vehicles: Two AVs with a human driven vehicle in between and to leverage the communication ability of the AVs to guide the whole system in an effective manner to a shared destination. A $3^{\text {rd }}$ strategy could be to use a $3^{\text {rd }}$-party application that leverages data available on Cloud from MMTS to develop an effective movement strategy for a platoon of AVs and legacy vehicles.

Table 5 A summary of identified key studies.

\begin{tabular}{ccll}
\hline S. no. & Year & \multicolumn{1}{c}{ Authors } & \multicolumn{1}{c}{ Title of the Paper } \\
\hline 1. & 2019 & $\begin{array}{l}\text { S Balaji, K Nathani } \\
\text { and R Santhakumar }\end{array}$ & $\begin{array}{l}\text { IoT technology, applications } \\
\text { and challenges: } \\
\text { A contemporary survey }\end{array}$ \\
2. 2020 & $\begin{array}{l}\text { D Minovski, C } \\
\text { Ahlund and K Mitra }\end{array}$ & $\begin{array}{l}\text { Modeling quality of IoT } \\
\text { experience in autonomous } \\
\text { vehicles }\end{array}$ \\
\hline
\end{tabular}

3. 2015 K Golestan, R Soua, F Situation awareness within Karray and MS Kamel the context of connected cars: A comprehensive review and recent trends

4. $2020 \quad$ P Kopelias, E

Connected \& autonomous Demiridi, K Vogiatzis, vehicles - Environmental A Skabardonis and V impacts - A review Zafiropoulou

5. 2020 KF Yuen, YD Wong, $\mathrm{F}$ Ma and $\mathrm{X}$ Wang

The determinants of public acceptance of autonomous vehicles: An innovation diffusion perspective

6. 2016 M Maurer, JC Gerdes, Autonomous driving: B Lenz and H Winner Technical, legal and social aspects

7. 2017 JE Siegel, DC Erb and A survey of the connected SE Sarma vehicle landscape Architectures, enabling technologies, applications, and development areas

8. 2019 F Arena and G Pau

An overview of vehicular communications

9. 2016 O Kaiwartya, AH

Internet of vehicles: Abdullah, Y Cao, A Motivation, layered Altameem, M Prasad, CT Lin and X Liu architecture, network model, challenges, and future aspects

Discusses quality of experience for human-AV interaction. Quality of IoT experience comprises quality of data, networks and context.

Identifies Client - Connection - Cloud architecture for IoV. Provides a comprehensive survey on situational awareness in connected cars.

Discusses how Cloud based CAVE can help in superior traffic management and reducing $\mathrm{CO}_{2}$ emissions.

Discusses the various constructs and measurements for the acceptance of AVs by general public.

Discusses implementation of human understood ethics into AV. Discusses process flow for control actions in AV.

Discusses technological challenges for application deployment in CAVE.

Discusses terminologies for interaction of an AV with its environment.

Discusses a 5 layered architecture for IoV. The 5 layers are 1) Perception layer, 2) Coordination layer, 3) Artificial intelligence layer, 4) Application layer and 5) Business layer. 


\begin{tabular}{ccll}
\hline S. no. & Year & \multicolumn{1}{c}{ Authors } & \multicolumn{1}{c}{ Title of the Paper } \\
\hline 10. & 2018 & $\begin{array}{l}\text { M Chen, Y Tian, G } \\
\text { Fortino, J Zhang and I } \\
\text { Humar }\end{array}$ & $\begin{array}{l}\text { Cognitive internet of } \\
\text { vehicles }\end{array}$ \\
11. & 2017 & $\begin{array}{l}\text { J Contreras-Castillo, S } \\
\text { Zeadally and JA } \\
\text { Guerrero-Ibanez }\end{array}$ & $\begin{array}{l}\text { Internet of vehicles: } \\
\text { Architecture, protocols, and } \\
\text { security }\end{array}$ \\
& &
\end{tabular}

12. 2015 JZ Varghese and RG Boone

13. 2016 M Realpe, BX Vintimilla and L Vlacic

14. 2020 H Zhou, W Xu, J Chen and $\mathrm{W}$ Wang

15. 2020 Z Ning, K Zhang, X Wang, L Guo, X Hu, J Huang, B Hu and RYK Kwok

16. 2018 A Hassani, PD Haghighi, S Ling, PP Jayaraman and A Zaslavsky

17. 2019 V Mannoni, V Berg, S Sesia and E Paraud

18. $2017 \mathrm{~J}$ Hu, S Chen, L Zhao, Y Li, J Fang, B Li and Y Shi

19. 2017 A Filippi, K Moerman, $\mathrm{V}$ Martinez, A Turley, O Haran and R Toledano

20. 2018 BMD Aragon, J Alonso-Zarate and A Laya

21. 2017 H Qiu, F Ahmad, R
Overview of autonomous vehicle sensors and systems

A Fault Tolerant Perception system for autonomous vehicles

Evolutionary V2X technologies toward the internet of vehicles: Challenges and opportunities

Intelligent edge computing in internet of vehicles: A joint computation offloading and caching solution

Querying IoT services: A smart carpark recommender use case

A comparison of the $\mathrm{V} 2 \mathrm{X}$ communication systems: ITS-G5 and C-V2X

Link level performance comparison between LTE V2X and DSRC

IEEE802.11p ahead of LTE$\mathrm{V} 2 \mathrm{~V}$ for safety applications

How connectivity is transforming the automotive ecosystem

Augmented vehicular
Relevant Discussion

Discusses a 5 layered architecture for IoV. The layers are named as 1) Sensing layer, 2) Communication layer, 3) Cognition layer, 4) Control layer and 5) Application layer.

Discusses a 7 layered architecture for IoV. The 7 layers are: 1) User-vehicle interface layer, 2) Data acquisition layer, 3) Data filtering and preprocessing layer, 4) Communication layer, 5) Control and management layer, 6) Processing layer and 7) Security layer.

Discusses the parameters that are desired for a sensor for its applicability in CAVE.

Presents a fault tolerant perception system for vision applications in AV.

Discusses challenges and opportunities relating to big-data driven $\mathrm{IoV}$ and Cloud/Edge based IoV.

Proposes an IoV architecture for CAVE powered by Edge.

Presents use case for Context as a Service for IoT based parking applications in AVs.

Refer Table 3 for comparison between 802.11p and LTE based wireless communication technology.

Refer Table 3 for comparison between 802.11p and LTE based wireless communication technology.

Refer Table 3 for comparison between 802.11p and LTE based wireless communication technology.

Refer Table 3 for comparison between 802.11p and LTE based wireless communication technology.

Discusses cooperative perception in 


\begin{tabular}{|c|c|c|c|c|}
\hline S. no. & Year & Authors & Title of the Paper & Relevant Discussion \\
\hline & & $\begin{array}{l}\text { Govindan, M } \\
\text { Gruteser, F Bai and G } \\
\text { Kar }\end{array}$ & $\begin{array}{l}\text { reality: Enabling extended } \\
\text { vision for future vehicles }\end{array}$ & $\begin{array}{l}\text { which an AV shares its visual } \\
\text { information with other AVs in CAVE } \\
\text { while preserving the respective } \\
\text { coordinate frames of reference. }\end{array}$ \\
\hline 22. & 2020 & $\begin{array}{l}\text { M Tsukada, T Oi, A } \\
\text { Ito, M Hirata and } \mathrm{H} \\
\text { Isaki }\end{array}$ & $\begin{array}{l}\text { AutoC2X: Open-source } \\
\text { software to realize V2X } \\
\text { cooperative perception } \\
\text { among autonomous vehicles }\end{array}$ & $\begin{array}{l}\text { Presents an open-source V2X } \\
\text { cooperative perception system that } \\
\text { integrates individual perception of an } \\
\text { AV with an open-source experimental } \\
\text { and prototyping software for } \\
\text { cooperative Intelligent Transport } \\
\text { Systems. }\end{array}$ \\
\hline 23. & 2017 & $\begin{array}{l}\text { X Wang, S Vozar and } \\
\text { E Olson }\end{array}$ & $\begin{array}{l}\text { Flag: Feature-based } \\
\text { localization between air and } \\
\text { ground }\end{array}$ & $\begin{array}{l}\text { Presents a method for localization by } \\
\text { comparing features observed on ground } \\
\text { to those that are observed through } \\
\text { aerial pictures. Compares the proposed } \\
\text { method with Vision based } \\
\text { Simultaneous Localization and } \\
\text { Mapping (V-SLAM). }\end{array}$ \\
\hline 24. & 2018 & $\begin{array}{l}\text { G Veneri and A } \\
\text { Capasso }\end{array}$ & $\begin{array}{l}\text { Hands-on industrial internet } \\
\text { of things: Create a powerful } \\
\text { industrial IoT infrastructure } \\
\text { using industry } 4.0\end{array}$ & $\begin{array}{l}\text { Discusses anomaly detection } \\
\text { methodologies for Industrial IoT. }\end{array}$ \\
\hline 25. & 2020 & $\begin{array}{l}\text { H Khayyam, B Javadi, } \\
\text { M Jalili and RN Jazar }\end{array}$ & $\begin{array}{l}\text { Artificial intelligence and } \\
\text { internet of things for } \\
\text { autonomous vehicles }\end{array}$ & $\begin{array}{l}\text { Discusses AI approaches and } \\
\text { challenges with deploying AI to AVs. } \\
\text { Discusses IoT, Cloud and Edge } \\
\text { technologies for AVs. }\end{array}$ \\
\hline 26. & 2016 & $\begin{array}{l}\text { A Lima, F Rocha, M } \\
\text { Volp and P Estives- } \\
\text { Verissimo }\end{array}$ & $\begin{array}{l}\text { Towards safe and secure } \\
\text { autonomous and cooperative } \\
\text { vehicle ecosystems }\end{array}$ & $\begin{array}{l}\text { Discusses threat model and vectors for } \\
\text { CAVE. }\end{array}$ \\
\hline
\end{tabular}

\section{Conclusions}

This paper reviews technologies and challenges for CAVE. Evolution of architectures paving way for CAVE are reviewed in the paper. The role that IoT technology plays for reliable perception of environment for an AV in CAVE is discussed. V2X communication is discussed and the 2 contending communication technologies: IEEE802.11p and LTE are reviewed and compared under various parameters for their usage in CAVE. The onboard diagnostics in AV is reduced to the problem of anomaly detection and is discussed as such. Control action strategies and threat vectors are reviewed for AVs in CAVE. A timeline that has transition from legacy vehicles to MMTS and from MMTS to CAVE is discussed. Strategies for effective movement in MMTS are presented. Table 5, that summarizes identified key studies is presented for the benefit of the readers. Future work may involve using various unsupervised anomaly detection methods to improve the proposed methodology for onboard diagnostics in an AV. Strategies presented for movement in MMTS may be implemented and compared through simulations. 


\section{References}

[1] Overview of Road Accidents in India, Available at: https://www.prsindia.org/policy/vital-stats/ overview-road-accidents-india, accessed September 2020.

[2] Self-Driving Cars Are Flummoxed by India's Chaotic Roads, Available at: https://www.thedrive.com /tech/12032/self-driving-cars-are-flummoxed-by-indias-chaotic-roads, accessed September 2020.

[3] S Balaji, K Nathani and R Santhakumar. IoT technology, applications and challenges: A contemporary survey. Wirel. Pers. Commun. 2019; 108, 363-88.

[4] D Minovski, C Ahlund and K Mitra. Modelling quality of IoT experience in autonomous vehicles. IEEE Internet Things J. 2020; 7, 3833-49.

[5] K Golestan, R Soua, F Karray and MS Kamel. Situation awareness within the context of connected cars: A comprehensive review and recent trends. Inf. Fusion 2016; 29, 68-83.

[6] P Kopelias, E Demiridi, K Vogiatzis, A Skabardonis and V Zafiropoulou. Connected \& autonomous vehicles - Environmental impacts - A review. Sci. Total Environ. 2020; 712, 135237.

[7] KF Yuen, YD Wong, F Ma and X Wang. The determinants of public acceptance of autonomous vehicles: An innovation diffusion perspective. J. Clean. Prod. 2020; 270, 121904.

[8] M Maurer, JC Gerdes, B Lenz and H Winner. Autonomous driving: Technical, legal and social aspects. Springer Nature, Berlin, Germany, 2016.

[9] JE Siegel, DC Erb and SE Sarma. A survey of the connected vehicle landscape - Architectures, enabling technologies, applications, and development areas. IEEE Trans. Intell. Transp. Syst. 2018; 19, 2391-406.

[10] F Arena and G Pau. An overview of vehicular communications. Future Internet 2019; 11, 27.

[11] O Kaiwartya, AH Abdullah, Y Cao, A Altameem, M Prasad, CT Lin and X Liu. Internet of vehicles: Motivation, layered architecture, network model, challenges, and future aspects. IEEE Access 2016; 4, 5356-73.

[12] M Chen, Y Tian, G Fortino, J Zhang and I Humar. Cognitive internet of vehicles. Comput. Commun. 2018; 120, 58-70.

[13] J Contreras-Castillo, S Zeadally and JA Guerrero-Ibanez. Internet of vehicles: Architecture, protocols, and security. IEEE Internet Things J. 2017; 5, 3701-9.

[14] Glossary of Sensor Terminology, Available at: https://onlinelibrary.wiley.com/doi/pdf/10.1002/978 1119069164.gloss, accessed October 2020.

[15] JZ Varghese and RG Boone. Overview of autonomous vehicle sensors and systems. In: Proceedings of the International Conference on Operations Excellence and Service Engineering, Orlando, Florida. 2015, p. 178-91.

[16] M Realpe, BX Vintimilla and L Vlacic. A Fault Tolerant Perception system for autonomous vehicles. In: Proceedings of the $35^{\text {th }}$ Chinese Control Conference, Chengdu, China. 2016, p. 6531-6.

[17] $\mathrm{H}$ Zhou, W Xu, J Chen and W Wang. Evolutionary V2X technologies toward the internet of vehicles: Challenges and opportunities. Proc. Inst. Electr. Electron. Eng. 2020; 108, 308-23.

[18] Z Ning, K Zhang, X Wang, L Guo, X Hu, J Huang, B Hu and RYK Kwok. Intelligent edge computing in internet of vehicles: A joint computation offloading and caching solution. IEEE Trans. Intell. Transp. Syst. 2020; 22, 2212-25.

[19] A Hassani, PD Haghighi, S Ling, PP Jayaraman and A Zaslavsky. Querying IoT services: A smart carpark recommender use case. In: Proceedings of the IEEE $4^{\text {th }}$ World Forum on Internet of Things, Singapore. 2018, p. 619-24.

[20] V Mannoni, V Berg, S Sesia and E Paraud. A comparison of the V2X communication systems: ITSG5 and C-V2X. In: Proceedings of the IEEE $89^{\text {th }}$ Vehicular Technology Conference, Kuala Lumpur, Malaysia. 2019, p. 1-5.

[21] J Hu, S Chen, L Zhao, Y Li, J Fang, B Li and Y Shi. Link level performance comparison between LTE V2X and DSRC. J. Commun. Inf. Netw. 2017; 2, 101-12.

[22] IEEE802.11p ahead of LTE-V2V for safety applications, Available at: https://www.nxp.com/docs/en /white -paper/LTE-V2V-WP.pdf, accessed May 2021.

[23] BMD Aragon, J Alonso-Zarate and A Laya. How connectivity is transforming the automotive ecosystem. Internet Technol. Lett. 2018; 1, e14.

[24] H Qiu, F Ahmad, R Govindan, M Gruteser, F Bai and G Kar. Augmented vehicular reality: Enabling extended vision for future vehicles. In: Proceedings of the $18^{\text {th }}$ International Workshop on Mobile Computing Systems and Applications, Sonoma, California. 2017, p. 67-72. 
[25] M Tsukada, T Oi, A Ito, M Hirata and H Isaki. AutoC2X: Open-source software to realize V2X cooperative perception among autonomous vehicles. In: Proceedings of the IEEE $92^{\text {nd }}$ Vehicular Technology Conference, Victoria, British Columbia, Canada. 2020, p. 1-6.

[26] X Wang, S Vozar and E Olson. Flag: Feature-based localization between air and ground. In: Proceedings of the IEEE International Conference on Robotics and Automation, Marina Bay Sands, Singapore. 2017, p. 3178-84.

[27] Multiple Kernel Anomaly Detection Algorithm, Available at https://ti.arc.nasa.gov/opensource/ projects/mkad, accessed May 2021.

[28] G Veneri and A Capasso. Hands-on industrial internet of things: Create a powerful industrial IoT infrastructure using industry 4.0. Packt Publishing, Birmingham, 2018.

[29] H Khayyam, B Javadi, M Jalili and RN Jazar. Artificial intelligence and internet of things for autonomous vehicles. In: RN Jazar and L Dai (Eds.). Nonlinear approaches in engineering applications. Springer, Cham, Switzerland, 2020, p. 39-68.

[30] A Lima, F Rocha, M Volp and P Estives-Verissimo. Towards safe and secure autonomous and cooperative vehicle ecosystems. In: Proceedings of the $2^{\text {nd }}$ ACM Workshop on Cyber-Physical Systems Security and Privacy, Vienna, Austria. 2016, p. 59-70. 